\title{
Tobacco Use, Cessation Research
}

National Cancer Institute

\section{Source}

National Cancer Institute. Tobacco Use, Cessation Research. NCI Thesaurus. Code C15991.

Studies to test and implement interventions among groups of individuals that are designed to stop current use of tobacco. (NCI/T RIP) 